\title{
Food insecurity among homeless and runaway adolescents
}

\author{
Les B Whitbeck ${ }^{1, *}$, Xiaojin Chen ${ }^{2}$ and Kurt D Johnson ${ }^{1}$ \\ 'University of Nebraska-Lincoln, Department of Sociology, 739 Oldfather Hall, Lincoln, NE 68588-0324, USA: \\ ${ }^{2}$ Department of Sociology, Tulane University, New Orleans, LA, USA
}

Submitted 17 May 2004: Accepted 19 May 2005

\begin{abstract}
Objective: The purpose of this study was to investigate the prevalence of food insecurity and factors related to it among homeless and runaway adolescents.

Design: Computer-assisted personal interviews were conducted with homeless and runaway adolescents directly on the streets and in shelters.

Setting: Interviews were conducted in eight Midwest cities: Des Moines, Cedar Rapids, Iowa City, Kansas City, Lincoln, Omaha, St. Louis and Wichita.

Subjects: The subjects were 428 (187 males; 241 females) homeless and runaway adolescents aged 16-19 years. Average age of the adolescents was 17.4 (standard deviation 1.05) years.

Results: About one-third of the adolescents had experienced food insecurity in the past 30 days. Factors associated with food insecurity were age of adolescent, a history of caretaker neglect and abuse, having ever spent time directly on the street, a small post-runaway social network, and engaging in deviant and non-deviant street foodacquisition strategies.

Conclusions: Based on these findings, our conservative estimate is that nationally more than 165000 homeless and runaway adolescents experienced food insecurity in the past 30 days. These adolescents are largely hidden from public notice and they are usually missed in studies that address national hunger.
\end{abstract}

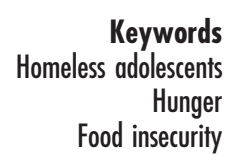

\begin{abstract}
Adolescent growth spurts are associated with voracious teen appetites and indiscriminate eating patterns. According to the Youth Risk Behavior Surveillance System conducted by the Centers for Disease Control and Prevention, the diets of adolescents often do not meet basic nutritional guidelines ${ }^{1,2}$. The erratic eating habits typical of adolescents and inconsistent food sources unique to being homeless combine to put runaway and homeless adolescents at significant risk for food insecurity and malnutrition, yet we know very little about nutrition among this hard-to-access population.

Most of the research dealing with nutrition and food insecurity among homeless people focuses on homeless adults or homeless families with children. We were able to locate only three recent studies that directly address food insecurity among homeless and runaway adolescents $^{3-5}$ and all of these were based on Canadian samples. This research indicated that street youth often experienced food insecurity due to lack of money. The precariousness of meeting basic food needs increased the likelihood of street youth turning to dangerous delinquent behaviours to subsist ${ }^{5}$. Institutional food sources did not fully address food insecurity due to barriers to utilisation such as perceived quality of food, rules for obtaining it, capacity, location of the food source, and mixing adults and youths.
\end{abstract}

The present paper reports an investigation of the prevalence of food insecurity and factors associated with it among runaway and homeless adolescents in small to moderate-sized Midwest cities. Our use of the term 'food insecurity' is based on the definition of the US Department of Agriculture (USDA): 'Limited or uncertain availability of nutritionally adequate and safe foods or limited or uncertain ability to acquire acceptable foods in socially dependable ways ${ }^{6}$. When living on the streets, homeless and runaway adolescents by definition meet these criteria for food insecurity. Although remarkably adaptive in securing food, during periods when they are unsheltered many resort to uncertain and risky subsistence strategies including panhandling for spare change, shop-lifting, dumpster diving, survival sex and theft ${ }^{5,7}$. Although there is substantial evidence that significant populations of runaways and street youth are present in small to moderatesized Midwest cities ${ }^{7}$, resources to address their health and nutritional needs often are limited or non-existent.

\section{Hypothesised correlates of food insecurity}

We hypothesised that factors associated with other healthrisk behaviours when on the streets would also be associated with food insecurity. For example, older adolescents, females and adolescents with samesex sexual orientation are at greater risk than their 
counterparts for street victimisation. Adolescents with family backgrounds of neglect and abuse and those who have spent time directly on the streets are also more likely to engage in deviant subsistence strategies when on the streets and to be victimised on the streets ${ }^{7}$. Both the proportion of the adolescent's social network from their home neighbourhood and the total number of people in their social networks are associated with tangible support $^{8}$. We expect that health-risk behaviours cluster such that adolescents with substance abuse problems will also be those who have nutritional deficits. Indeed, the more the adolescent is immersed in the street culture, the more likely he or she will experience food insecurity.

\section{Method}

\section{Sample}

Participants were recruited as part of a 3-year longitudinal study of homeless and runaway youth in the Midwest. The data reported here are from the baseline interview. The respondents were interviewed by full-time specially trained street interviewers directly on the streets and in shelters in eight Midwest cities (St. Louis, Kansas City, Omaha, Lincoln, Des Moines, Cedar Rapids, Iowa City and Wichita). To be eligible to participate, the young person had to be between the ages of 16 and 19 years and homeless. Our definition of 'homeless' was that the adolescent must be residing in a shelter, on the street or living independently (e.g. friends, transitional living) because they had run away, been pushed out or drifted out of their family of origin. Based on interviewer reports, approximately $90 \%$ of the 505 homeless and runaway adolescents who were approached for an initial interview and who met study criteria agreed to participate. Of the 455 respondents who completed the first baseline interview, $94.1 \%$ or 428 ( 187 males; 241 females) completed the second baseline diagnostic interview. Non-completers had a statistically significant higher age when they first ran away (14.84 vs. 13.41 years, $P \leq 0.05$ ). They were more likely to report that they were heterosexual (100\% vs. $85 \%$ of completers) and less likely to report having been physically victimised when on their own than were completers.

The adolescents were informed that this was a longitudinal study and the tracking protocols were explained. Informed consent was a two-stage process. First, the study was explained and informed consent was obtained from the adolescent. They were assured that refusal to participate in the study, refusal of any question or stopping the interview process would have no effect on current or future services provided. Second, all adolescents were asked if we could contact their parents. If permission was granted, parents were contacted and informed consent to talk to a minor less than 18 years old was obtained verbally. The parents also were asked to participate in a computer-assisted telephone interview.
Results from the parent interviews are not discussed in this paper. If the adolescent was in a shelter, we followed shelter policies of parental permission. In the few cases where the adolescent was less than 18 years old, not in a shelter and refused permission to contact parents, he/she was treated as an emancipated minor in accordance with National Institutes of Health guidelines?. The consent process and questionnaires were approved by the University of Nebraska-Lincoln Institutional Review Board. A National Institute of Mental Health Certificate of Confidentiality was obtained to protect the respondents' statements regarding potentially illegal activities (e.g. drug use).

The street interviewers underwent two weeks of intensive training regarding computer-assisted personal interviewing procedures and administering the four UM-CIDI (major depressive episodes, post-traumatic stress disorder, alcohol use/abuse, and drug use/abuse) and one DISC-R (conduct disorder) indices. They then returned to their shelters and administered several 'practice' interviews with staff and respondents 20 years or older. After completing their practice interviews the interviewers returned to the university for a second week of training. All interviews were conducted on laptop computers and downloaded electronically to a special secure university server.

We designed a sampling strategy for the current study that incorporated sampling units of fixed and natural sites similar to the design used by Kipke et al. in their Los Angeles study of homeless youth with a year-long window of sampling to capture the time dimensions ${ }^{10}$. The sampling design involved repeatedly checking locations where homeless youth were likely to be found in each of the target cities. Locations included shelters and outreach programmes serving homeless youth, drop-in centres and various 'street' locations where young homeless people were most likely to be located. Research has demonstrated that using sampling designs that involve multiple points of entry to homeless populations are most effective in generating a diverse sample ${ }^{11,12}$. The interviewers all had prior experience in their respective cities as youth outreach workers and brought considerable knowledge regarding optimal areas of the city for locating youth on their own. The sampling protocol included going to these locations in the cities at varying times of the day, on both weekday and weekends, over the course of 12 months. Since episodes of homelessness are of varying duration, a 1-year time frame provided an increased probability of capturing youth who have short-term exposure to homelessness. The interviewers were told to continue recruiting until their caseload reached 60 adolescents, whom they would then track and re-interview at 3-month intervals.

The first-wave interview was in two parts. The first consisted of a social history and symptom scales. The respondent was then asked to meet for a second interview 
during which the diagnostic interviews were conducted. These two interviews made up the baseline assessment for the study and usually were completed within one or two days, so that no significant time lapsed between the first part of the baseline interview and the second diagnostic interview. The respondents were paid $\$ 25$ for the first interview and $\$ 25$ for the second.

\section{Measures}

Age of adolescent at time of interview was calculated using the date of birth of the respondent and the date of the baseline interview. Gender of adolescent was coded 0 for females and 1 for males (56\% vs. 44\%, respectively).

Adolescent sexual orientation was assessed by a question in which the adolescents identified themselves as straight, heterosexual, gay/lesbian, bisexual, never thought about it, something else, or confused or unsure. The variable was recoded so that any individual listing a non-heterosexual or unsure sexual identity was coded as non-heterosexual. Response categories were 1 for heterosexual and 0 for non-heterosexual.

Caretaker abuse/neglect was measured with a 13-item scale that asked adolescents how often a parent or adult caretaker who was supposed to be taking care of them ever punished them by making them go a full day without food or water, abandoned them for at least $24 \mathrm{~h}$, threw something at them in anger, pushed them, slapped them, hit them with an object, beat them up with their fists, threatened or assaulted them with a weapon ${ }^{13}$ and/or sexually abused them. Because an unequal number of neglect, physical abuse and sexual abuse items were asked, items were weighted to give equal importance to the three indicators in the summed scale. Scale scores were coded such that the higher the score, the higher the rate of abuse. Cronbach's $\alpha$ for caretaker abuse/neglect was 0.94 .

Adolescents were asked if they had ever spent one or more nights on the street, in an abandoned building or another place out in the open. Those individuals who had not spent at least one night on the street were coded as 0 . Approximately $49 \%$ of the sample had spent at least one night on the street.

Proportion of social network members from 'bome' refers to a count of family members, friends and relatives in the adolescents' social networks who were from their home (pre-runaway) neighbourhood.

Total number in social network was the total number of persons reported by the adolescent who provided him or her with instrumental support such as food, money or shelter.

Substance abuse was assessed by meeting lifetime criteria for either alcohol or drug abuse. The University of Michigan Composite International Diagnostic Interview (UM-CIDI) was used to assess alcohol abuse and drug abuse. The UM-CIDI is based on criteria of the third revised edition of the Diagnostic and Statistical Manual of Mental Disorders (DSM-III-R) and represents the University of Michigan revision of the CID $^{14}$ used in the National Comorbidity Survey ${ }^{15,16}$. The UM-CIDI is a wellestablished diagnostic instrument ${ }^{14}$ that has shown excellent inter-rater reliability, test-retest reliability and validity for the five diagnoses that were used in this study ${ }^{17}$. The UM-CIDI is currently the state-of-the-art diagnostic interview schedule that has been used extensively with trained interviewers who are not clinicians.

Deviant food strategies were measured by four items asking adolescents how they got food while they were on the street. These strategies included panhandling or spare changing; stealing or shop-lifting; prostitution; and using dumpsters. A count procedure was used to compute the composite scale. If the adolescent had used the strategy the item was given a score of 1 ; if not, it was coded 0 . The range was from 0 to 4 .

Non-deviant food strategies were assessed by five items in which the adolescents were asked whether they had used strategies of buying food, getting food from parents or caretakers, getting it from friends, getting food from social services or using state vouchers. A count procedure was used to compute the composite scale. If the adolescent had used the strategy the item was given a score of 1 ; if not, it was coded 0 . The range was from 0 to 5 .

We assessed food insecurity with three items adapted from the USDA food insecurity scale ${ }^{6}$ that dealt specifically with going without or cutting back food. It should be noted that these are only three items from a 16-item scale and they do not reflect the nuances of the full USDA measure. The intent in adapting this measure was to capture actual self-reported hunger for adolescents when they were on their own. Therefore we selected items that specifically addressed going without food. The modifications consisted of deleting the words 'or other adults' from items 8-10 of the 1st Level Internal Screen Scale and changing the time addressed by the questions from 12 months to 30 days to capture the immediacy of need (see Guide to Measuring Household Food Insecurity, p. 24) ${ }^{6}$. The change to a 30-day time period is listed as an acceptable modification of the measure (see Guide to Measuring Housebold Food Insecurity, p. 25) ${ }^{6}$. The adolescents were asked if in the last 30 days they had cut the size of meals or skipped meals, if they had not eaten for a whole day, and if they had been hungry but couldn't eat because they did not have money to buy food. Response categories were $0=$ not in the last 30 days, $1=$ once, $2=$ a few times and $3=$ many times. The three-item measure was summed. Cronbach's $\alpha$ for this scale was 0.89 .

Table 1 reports the descriptive statistics for all variables used in the analyses.

\section{Results}

The adolescents ranged in age from 16 to 19 years with an average age of 17.4 (SD 1.05) years. Fifty-nine per cent 
Table 1 Descriptive statistics for variables used in the analysis $(n=428)$

\begin{tabular}{lcccl}
\hline Variable & Minimum & Maximum & Mean & SD \\
\hline Age of adolescent & 16 & 19 & 17.38 & 1.05 \\
$\begin{array}{l}\text { Sex of adolescent } \\
\quad(1=\text { male) }\end{array}$ & 0 & 1 & 0.44 & 0.5 \\
$\quad \begin{array}{lllll}\text { Sexual orientation } \\
\quad(1=\text { heterosexual) }\end{array}$ & 0 & 1 & 0.85 & 0.35 \\
$\quad$ Caretaker abuse/neglect & 0 & 3.85 & 1.38 & 0.78 \\
Ever on the street & 0 & 1 & 0.49 & 0.5 \\
Proportion of social network & 0 & 1 & 0.55 & 0.44 \\
$\quad$ members from home & & & & \\
$\quad$ Total number in social network & 0 & 19 & 3.74 & 4.21 \\
$\quad$ Substance abuse in past year & 0 & 1 & 0.44 & 0.5 \\
Deviant food strategies & 0 & 4 & 0.36 & 0.71 \\
Non-deviant food strategies & 0 & 5 & 2.63 & 1.04 \\
Food insecurity & 0 & 9 & 2.36 & 3.18 \\
$\quad$ three-item measure) & & & & \\
\hline
\end{tabular}

SD - standard deviation.

were European American, 22\% were non-Hispanic African American and 5\% were Hispanic, with the remaining selfidentified as American Indian, Asian or Pacific Islander, or bi-racial. Fifteen per cent identified themselves as gay, lesbian or bisexual. Sixty-two per cent of the adolescents reported that the population of their city of origin was 100000 or greater, $10 \%$ said they were from a suburb of a large city, 8\% were from a medium-sized city with $50000-$ 100000 inhabitants, 8\% were from a small city of $10000-$ 50000 inhabitants, and 12\% were from small towns or rural communities of 10000 or fewer inhabitants.

Table 2 indicates that approximately one-third of the adolescents reported food insecurity during the 30 days prior to the interview (i.e. those reporting cutting or skipping meals, ever having gone without food a whole day, or going hungry; range 29.44-35.83\%). Twenty-five per cent had gone a whole day without eating at least 'a few times' in the last 30 days. Twenty-one per cent had cut the size of meals or skipped meals 'many times' and 13\% had done so 'a few times' in the past 30 days. Fourteen per cent of the adolescents had gone without food when they were hungry 'many times' in the past 30 days due to lack of funds to buy food. An additional 17\% had gone hungry 'a few times' in the past 30 days because they had no money to buy food.

We used stepwise ordinary least-squares regression with list-wise deletion of missing data to investigate factors associated with food insecurity on the streets (Table 3). The outcome variable was our three-item measure of going hungry in the past 30 days. Control variables, age, gender and sexual orientation of the adolescents were first entered into the equation in Model 1. In Models 2 and 3, variables that pertained to the runaway adolescent's history at home (neglect and/abuse by caretakers) and on the street (ever on street) were added to the equation. Social network variables were added in Models 4 and 5 to assess availability of social support as a factor in obtaining food. The degree of substance abuse was added to the equation to investigate the potential negative effects of use and acquiring alcohol and drugs on food acquisition. After controlling for all of these factors that potentially influence food acquisition, we added in actual food-acquisition strategies to Model 6.

Among our control variables (Model 1), older adolescents and those with same-sex orientation were those most likely to report food insecurity. When a history of caretaker abuse and/or neglect was added in Model 2, it was positively associated with food insecurity, gender (being male) became statistically significant and sexual orientation lost significance. In Model 3, having ever spent time directly on the streets was introduced and it was positively associated with food insecurity; gender became non-significant in Model 3. The social network variables were introduced in Model 4. The proportion of the adolescents' social network from home was non-significant; however, the size of the social network was negatively related to food insecurity. The larger the social network, the less likely the adolescent would experience food insecurity. Substance abuse (meeting UM-CIDI diagnostic criteria for alcohol or drug abuse) was added in Model 5 and it was positively associated with food insecurity. Use of deviant and non-deviant foodacquisition strategies was positively associated with food insecurity in Model 6. Substance abuse lost significance when food strategies were added into the model.

In summary, in the final model (Model 6), age, a history of caretaker abuse and/or neglect, having ever spent time directly on the streets, being part of large social network, and having deviant and non-deviant food-acquisition strategies when the adolescents were on their own were associated with food insecurity. The final model explained $35 \%$ of the variance of food insecurity.

\section{Discussion}

The findings indicate that about one-third of the adolescents interviewed responded positively to at

Table 2 Food insecurity (\%, three-item measure) in past 30 days among homeless and runaway adolescents $(n=428)$

\begin{tabular}{llccr}
\hline & Never & Once & A few times & Many times \\
\hline Cut the size of meals or skipped meals & 64.17 & 1.17 & 13.35 & 21.31 \\
Did not eat for a whole day & 70.56 & 4.44 & 14.25 & 10.75 \\
Was hungry but couldn't buy food due to & 65.89 & 2.80 & 17.29 & 14.02 \\
$\quad$ lack of money & & & & \\
\hline
\end{tabular}


Table 3 Multiple regression model ( $\beta$ coefficients and explained variance, $R^{2}$ ) predicting food insecurity (three-item measure) among homeless and runaway adolescents $(n=425)$

\begin{tabular}{|c|c|c|c|c|c|c|}
\hline & Model 1 & Model 2 & Model 3 & Model 4 & Model 5 & Model 6 \\
\hline Age & $0.29^{\star *}$ & $0.25^{\star \star}$ & $0.19^{\star \star}$ & $0.16^{\star \star}$ & $0.16^{\star \star}$ & $0.17^{\star \star}$ \\
\hline Male & 0.08 & $0.10^{*}$ & 0.06 & 0.07 & 0.06 & 0.03 \\
\hline Heterosexual & $-0.10^{\star}$ & -0.08 & -0.06 & -0.08 & -0.07 & -0.04 \\
\hline Caretaker abuse/neglect & & $0.29^{\star \star}$ & $0.24^{\star *}$ & $0.21^{\star \star}$ & $0.20^{\star \star}$ & $0.18^{\star \star}$ \\
\hline Ever on the street & & & $0.22^{\star \star}$ & $0.22^{\star *}$ & $0.21^{\star \star}$ & $0.13^{\star \star}$ \\
\hline Proportion of social network members from home & & & & -0.03 & -0.03 & 0.01 \\
\hline Total number in social network & & & & $-0.14^{\star \star}$ & $-0.14^{\star *}$ & $-0.14^{\star *}$ \\
\hline Substance abuse & & & & & $0.10^{*}$ & 0.07 \\
\hline Deviant food strategies & & & & & & $0.31^{\star *}$ \\
\hline Non-deviant food strategies & & & & & & $0.09^{\star}$ \\
\hline$R^{2}$ & 0.11 & 0.20 & 0.23 & 0.25 & 0.26 & 0.35 \\
\hline
\end{tabular}

${ }^{*}, P<0.05 ;{ }^{* *}, P<0.01$

least one of the food insecurity items (i.e. cutting or skipping meals, not eating for a whole day and/or reporting hunger because they could not afford food) at least 'a few times' in the past 30 days. Twenty-five per cent reported that they had gone a whole day without food at least 'a few times' in the past 30 days. Insufficient food can have significant health implications during adolescence, including critical developmental consequences and reducing the effectiveness of the immune system in resisting potential infections.

Those most apt to experience food insecurity were those most vulnerable to health stressors in other areas of their lives. They were more likely to be experiencing stress originating from caretaker neglect and abuse, more likely to have spent time directly on the street, more likely to be substance abusers, and more likely to be isolated (e.g. have small social networks). They were adapting to life on their own by creating independent food-acquisition strategies. Some of these involved legitimate food sources such as borrowing money, getting money or food from relatives or from social welfare resources. Other strategies involved panhandling, theft and survival sex, all of which are associated with risk for victimisation ${ }^{18,19}$. Two important resiliency factors stand out: the number of people in their social networks and the availability of non-deviant food strategies. The size of social networks was negatively related to food insecurity probably because of sharing and borrowing strategies. Although non-deviant foodacquisition strategies were positively related to food insecurity - that is, the adolescents who used them were going hungry - they indicated the adolescents were engaging in low-risk food strategies.

\section{Limitations}

This research has several important limitations that should be noted. Perhaps most important is the three-item scale of food insecurity. There are several limitations associated with measuring food insecurity with the entire household scale (see p. 16 of the Guide to Measuring Food Insecurity) ${ }^{6}$. The scale in its entirety does not capture all aspects of food insecurity. Certainly our three-item adaptation does not do so.

That the sample is limited to small to moderate-sized cities in four Midwest states may be both a strength and a limitation of the study. It is a strength in that it documents the presence and plight of runaways in cities where they are often unacknowledged. The limitation is that the results may not be generalisable to other geographic areas or to larger metropolitan areas. Also, all of our measures are self-reports and reflect the potential biases associated with adolescent reports of health conditions and concerns. Our expectation based on our interviewers' assessments is that nutritional problems are probably underreported by the adolescents.

\section{Conclusions}

We know too little about the nutritional needs of runaway and homeless adolescents. Although national estimates have become dated and we have no comprehensive studies of the number of homeless adolescents in the $\mathrm{USA}^{20}$, the best available estimates place the number at about $500000^{21,22}$. Given what may be conservative national estimates of the number of homeless adolescents nationally, and what are probably conservative estimates of food insecurity rates based on a sample from small to moderate-sized Midwest cities, about one-third or approximately 165000 homeless and runaway adolescents went hungry (e.g. cut or missed meals, did not eat for a whole day and/or were hungry because they couldn't afford food) in the past 30 days. These adolescents are largely hidden from others and ignored by government policy-makers. They are missed in studies that address national hunger, yet they comprise one of the most nutritionally at-risk categories of children in the nation.

Food insecurity is but one of a myriad of health risks faced by runaway and homeless adolescents; however, it is a major developmental concern. Small and moderatesized cities often do not even acknowledge the presence of street youth in their communities let alone provide outreach and specialised food programmes. However, the 
problem of food insecurity could be addressed by innovative food outreach programmes that take food directly to the streets where the adolescents hang out or by supplying attractive, age-appropriate food programmes in drop-in centres. Some moderate-sized cities such as Des Moines have street outreach vans that supply sandwiches and other necessities directly on the streets. Others have drop-in centres specifically for young people that provide hot meals.

Over the past two decades, our society has come to accept homeless adults as part of the urban landscape. Although once a societal concern, recently homeless adolescents have dropped from public and policy attention. Our national nutrition policy-makers should include this significant population of young people in their strategies to eliminate hunger in our country.

\section{Acknowledgement}

This research was funded by the National Institute of Mental Health (MH 57110) (Les Whitbeck, Principal Investigator).

\section{References}

1 Kann L, Kinchen SA, Williams BL, Ross JG, Lowry R, Grunbaum JA, et al. Youth Risk Behavior Surveillance United States, 1999. Morbidity and Mortality Weekly Report. CDC Surveillance Summaries 2000; 49: 1-32.

2 Grunbaum JA, Kann L, Kinchen SA, Ross R, Hawkins J, Lowry R, et al. Youth Risk Behavior Surveillance United States, 2003. Morbidity and Mortality Weekly Report. CDC Surveillance Summaries 2004; 53: 1-96.

3 Dachner N, Tarasuk V. Homeless 'squeegee' kids: food insecurity and daily survival. Social Science \& Medicine 2002; 54: 1039-49.

4 Antonaides M, Tarasuk V. A survey of food problems experienced by Toronto street youth. Canadian Journal of Public Health 1998; 89: 371-5.

5 McCarthy B, Hagen J. Surviving on the street: the experiences of homeless youth. Journal of Adolescent Research 1992; 7: 412-30.

6 Bickel G, Nord M, Price C, Hamilton W, Cook J. Guide to Measuring Housebold Food Security, Revised 2000. Alexandria, VA: US Department of Agriculture, Food and Nutrition Services, 2000.

7 Whitbeck L, Hoyt D. Nowhere to Grow: Homeless and Runaway Adolescents and Their Families. New York: Aldine de Gruyter, 1999.
8 Johnson KD, Whitbeck LB, Hoyt DR. Predictors of social network composition among homeless and runaway adolescents. Journal of Adolescence 2005; 28: 231-48.

9 Department of Health and Human Services. Protection of Human Subjects, Code of Federal Regulations, Title 45, Public Welfare, Department of Health and Human Services, National Institutes of Health, Office for Protection from Research Risks, Part 46. Revised 13 November 2001.

10 Kipke MD, O'Connor S, Nelson B, Anderson J. A probability sampling for assessing the effectiveness of outreach for street youth. In: Greenberg JB, Neumann MS, eds. What We Have Learned From the AIDS Evaluation of Street Outreach Projects, A Summary Document. Atlanta, GA: Department of Health and Human Services, Centers for Disease Control and Prevention, 2000.

11 Burt MA. Practical Methods for Counting Homeless People: A Manual for State and Local Jurisdictions, 2nd ed. Washington, DC: Urban Institute, 1996.

12 Koegel P, Burnam M, Morton J. Enumerating homeless people: alternative strategies and their consequences. Evaluation Review 1996; 20: 378-403.

13 Straus M, Gelles R. Physical Violence in American Families. Brunswick, NJ: Transaction Books, 1990.

14 World Health Organization (WHO). Composite International Diagnostic Interview (CIDI), Version 1.0. Geneva: WHO, 1990.

15 Kessler R. The National Comorbidity Survey of the United States. International Review of Psychiatry 1994; 6: 365-76.

16 Kessler R. Building on the ECA: The National Comorbidity Survey and the Children's ECA. International Journal of Methods in Psychiatric Research 1994; 4: 81-94.

17 Wittchen H. Reliability and validity studies of the WHOComposite International Diagnostic Interview (CIDI): a critical review. Journal of Psychiatric Research 1994; 28: 57-84.

18 Tyler K, Whitbeck L, Hoyt D, Johnson K. Self-mutilation and homeless youth: the role of family abuse, street experiences, and mental disorders. Journal of Research on Adolescence 2003; 13: 457-74.

19 Whitbeck L, Hoyt D, Yoder K, Cauce AM, Paradise M. Deviant behavior and victimization among homeless and runaway adolescents. Journal of Interpersonal Violence 2001; 16: 1175-2104.

20 Rotheram-Borus MJ, Parra M, Cantwell C, Gwadz M, Murphy DA. Runaway and homeless youths. In: DiClemente R, Hansen W, Ponton L, eds. Handbook of Adolescent Risk Behavior. New York: Plenum Press, 2001; 369-91.

21 House Committee on Education and Labor, Subcommittee on Human Resources. Juvenile Justice, Runaway Youth, and Missing Children's Act, amendments, 98th Congress, 2nd Session, 7 March, Y4, E8, 1:j98/15; 1984.

22 Office of Juvenile Justice Delinquency Prevention (OJJDP). Missing, Abducted, Runaway and Throwaway Children in America, First Report: Numbers and Characteristics, National Incidence Studies. Washington, DC: OJJDP, US Department of Justice, 1990. 